\title{
First Space Cohomology of the Orthosymplectic Lie Superalgebra in the Lie Superalgebra of Superpseudodifferential Operators
}

\section{Imed Basdouri}

Received: 8 November 2010 / Accepted: 30 May 2011 / Published online: 30 June 2011

(C) The Author(s) 2011. This article is published with open access at Springerlink.com

\begin{abstract}
We investigate the first cohomology space associated with the embedding of the Lie Orthosymplectic superalgebra $\mathfrak{o s p}(n \mid 2)$ on the $(1, \mathrm{n})$-dimensional superspace $\mathbb{R}^{1 \mid n}$ in the Lie superalgebra $\mathcal{S} \Psi \mathcal{D O}(n)$ of superpseudodifferential operators with smooth coefficients, where $n=0,1,2$. Following Ovsienko and Roger, we give erxplicit expressions of the basis cocycles.
\end{abstract}

Keywords Cohomology - Orthosymplectic superalgebra • Superpseudodifferential operators $\cdot$ Poisson superalgebra

Mathematics Subject Classification (2010) 53 D 55

\section{Introduction}

Let $\mathbb{R}^{1 \mid n}$ be the superspace with coordinates $\left(x, \theta_{1}, \ldots, \theta_{n}\right)$, where $x$ is an even indeterminate and $\theta_{1}, \ldots, \theta_{n}$ are odd indeterminates: $\theta_{i} \theta_{j}=-\theta_{j} \theta_{i}$. This superspace is equipped with the standard contact structure given by the distribution $D=$ $\left\langle\bar{\eta}_{1}, \ldots, \bar{\eta}_{n}\right\rangle$ generated by the vector fields $\bar{\eta}_{i}=\partial_{\theta_{i}}-\theta_{i} \partial_{x}$. That is, the distribution $D$ is the kernel of the following 1-form:

$$
\alpha_{n}=d x+\sum_{i=1}^{n} \theta_{i} d \theta_{i} .
$$

Presented by Alain Verschoren.

I. Basdouri $(\bowtie)$

Département de Mathématiques, Faculté des Sciences de Gafsa Tunisie,

Zarroug 2112, Gafsa, Tunisia

e-mail: basdourimed@yahoo.fr 
Consider the superspace $C^{\infty}\left(\mathbb{R}^{1 \mid n}\right)$ which is the space of functions $F$ of the form:

$$
F=\sum_{1 \leq i_{1}<\cdots<i_{k} \leq n} f_{i_{1}, \ldots, i_{k}}(x) \theta_{i_{1}} \cdots \theta_{i_{k}} \quad \text { where } \quad f_{i_{1}, \ldots, i_{k}} \in C^{\infty}(\mathbb{R}) .
$$

Of course, even (respectively odd) elements in $C^{\infty}\left(\mathbb{R}^{1 \mid n}\right)$ are the functions (1.1) for which the summation is only over even (respectively odd) integer $k$. Note $p(F)$ the parity of a homogeneous function $F$. On $C^{\infty}\left(\mathbb{R}^{1 \mid n}\right)$, we consider the contact bracket

$$
\langle F, G\rangle=F G^{\prime}-F^{\prime} G-\frac{1}{2}(-1)^{p(F)} \sum_{i=1}^{n} \bar{\eta}_{i}(F) \bar{\eta}_{i}(G)
$$

where the superscript 'stands for $\frac{\partial}{\partial x}$. Consider the superspace $\mathcal{K}(n)$ of contact vector fields on $\mathbb{R}^{1 \mid n}$. That is, $\mathcal{K}(n)$ is the superspace of vector fields on $\mathbb{R}^{1 \mid n}$ preserving the distribution $\left\langle\bar{\eta}_{1}, \ldots, \bar{\eta}_{n}\right\rangle$ :

$$
\mathcal{K}(n)=\left\{X \in \operatorname{Vect}\left(\mathbb{R}^{1 \mid n}\right) \mid\left[X, \bar{\eta}_{i}\right]=F_{X} \bar{\eta}_{i} \quad \text { for some } F_{X} \in C^{\infty}\left(\mathbb{R}^{1 \mid n}\right)\right\} .
$$

The Lie superalgebra $\mathcal{K}(n)$ is spanned by the vector fields of the form:

$$
X_{F}=F \partial_{x}-\frac{1}{2}(-1)^{p(F)} \sum_{i=1}^{n} \bar{\eta}_{i}(F) \bar{\eta}_{i}, \quad \text { where } \quad F \in C^{\infty}\left(\mathbb{R}^{1 \mid n}\right) .
$$

The vector field $X_{F}$ has the same parity as $F$. The bracket in $\mathcal{K}(n)$ can be written as:

$$
\left[X_{F}, X_{G}\right]=X_{\langle F, G\rangle} \text {. }
$$

Now, consider the 1-parameter action of $\mathcal{K}(n)$ on $C^{\infty}\left(\mathbb{R}^{1 \mid n}\right)$ given by the rule:

$$
\mathfrak{L}_{X_{F}}^{\lambda}=X_{F}+\lambda F^{\prime} .
$$

We denote this $\mathcal{K}(n)$-module by $\mathfrak{F}_{\lambda}^{n}$, the space of all weighted densities on $\mathbb{R}^{1 \mid n}$ of weight $\lambda$ :

$$
\mathfrak{F}_{\lambda}^{n}=\left\{F \alpha_{n}^{\lambda} \mid F \in C^{\infty}\left(\mathbb{R}^{1 \mid n}\right)\right\}
$$

The superspace of the supercommutative algebra of superpseudodifferential symbols on $\mathbb{R}^{1 \mid n}$ with its natural multiplication is spanned by the series

$$
\mathcal{S P}(n)=\left\{F=\sum_{k \geq-M} \sum_{\epsilon=\left(\epsilon_{1}, \ldots, \epsilon_{n}\right)} a_{k, \epsilon}(x, \theta) \xi^{-k} \bar{\theta}_{1}{ }^{\epsilon_{1}} \ldots \bar{\theta}_{n}^{\epsilon_{n}}: a_{k, \epsilon} \in C^{\infty}\left(\mathbb{R}^{1 \mid n}\right) ; \epsilon_{i}=0,1 ; M \in \mathbb{N}\right\},
$$

where $\xi$ corresponds to $\partial_{x}$ and $\bar{\theta}_{i}$ corresponds to $\partial_{\theta_{i}}\left(p\left(\bar{\theta}_{i}\right)=1\right)$.

The space $\mathcal{S P}(n)$ has a structure of the Poisson Lie superalgebra given by the following bracket :

$$
\{F, G\}=\frac{\partial(F)}{\partial \xi} \frac{\partial(G)}{\partial x}-\frac{\partial(F)}{\partial x} \frac{\partial(G)}{\partial \xi}-(-1)^{p(F)} \sum_{i=1}^{n}\left(\frac{\partial(F)}{\partial \theta_{i}} \frac{\partial(G)}{\partial \bar{\theta}_{i}}+\frac{\partial(F)}{\partial \bar{\theta}_{i}} \frac{\partial(G)}{\partial \theta_{i}}\right) .
$$


The associative superalgebra of superpseudodifferential operators $\mathcal{S} \Psi \mathcal{D O}(n)$ over $\mathbb{R}^{1 \mid n}$ has the same underlying vector space as $\mathcal{S P}(n)$ but the multiplication is now defined by the following rule:

$$
F \circ G=\sum_{k \geq 0, v_{i}=0,1} \frac{(-1)^{p(F)+1}}{k !}\left(\partial_{\xi}^{k} \partial_{\bar{\theta}_{i}}^{v_{i}} F\right)\left(\partial_{x}^{k} \partial_{\theta_{i}}^{v_{i}} G\right) .
$$

This composition rule induces the supercommutator defined by:

$$
[F, G]=F \circ G-(-1)^{p(F) p(G)} G \circ F .
$$

Ovsienko and Roger [10] calculated the space $\mathrm{H}_{\text {diff }}^{1}\left(\operatorname{vect}\left(S^{1}\right), \Psi \mathcal{D O}\right)$. They used the results of Fuchs [7] on $\mathrm{H}_{\text {diff }}^{1}\left(\mathfrak{v e c t}\left(S^{1}\right), \mathcal{F}_{\lambda}\right)$. To compute $\mathrm{H}_{\text {diff }}^{1}\left(\mathfrak{v e c t}\left(S^{1}\right), \Psi \mathcal{D O}\right)$ Ovsienko and Roger applied the theory of spectral seqences to a filtered module over a Lie algebra.

In paper [1,2], using the same methods as in the paper [10] the authors computed $\mathrm{H}_{\text {diff }}^{1}(\mathcal{K}(1), \mathcal{S} \Psi \mathcal{D O}(1))$ and $\mathrm{H}_{\text {diff }}^{1}(\mathcal{K}(2), \mathcal{S} \Psi \mathcal{D O}(2))$.

In this paper, we restrict ourselves to the cases $n=0,1,2$ and we restrict the action to the orthosymplectic Lie (super)algebra $\mathfrak{o s p}(n \mid 2)$ and we consider the spaces $\mathcal{S P}(n)$ as $\mathfrak{o s p}(n \mid 2)$-modules. Of course, the case $n=0$ corresponds to the classical setting: $\mathcal{K}(0)=\mathfrak{v e c t}(\mathbb{R})$ and the corresponding orthosymplectic Lie algebra osp $(0 \mid 2)$ is nothing but the classical Lie algebra $\mathfrak{s l}(2)$ which is isomorphic to the Lie subalgebra of $\mathfrak{v e c t}(\mathbb{R})$ generated by

$$
\mathfrak{s l}(2)=\operatorname{Span}\left(\frac{d}{d x}, x \frac{d}{d x}, x^{2} \frac{d}{d x}\right),
$$

$\mathcal{S P}(0)$ is the classical spaces of symbols, usually denoted

$$
\mathcal{P}=\left\{F(x, \xi), F(x, \xi)=\sum_{-\infty}^{m} f_{k}(x) \xi^{k}\right\} .
$$

and $\mathcal{S} \Psi \mathcal{D O}(0)$ is the classical associative algebra of pseudodifferential operators, usually denoted $\Psi \mathcal{D O}$.

The Lie superalgebra osp $(1 \mid 2)$ can realized as a subalgebra of $\mathcal{K}(1)$ :

$$
\mathfrak{o s p}(1 \mid 2)=\operatorname{Span}\left(X_{1}, X_{x}, X_{x^{2}}, X_{x \theta_{1}}, X_{\theta_{1}}\right) .
$$

While, the Lie superalgebra osp (2|2) is realized as a subalgebra of $\mathcal{K}(2)$ :

$$
\mathfrak{o s p}(2 \mid 2)=\operatorname{Span}\left(X_{1}, X_{x}, X_{x^{2}}, X_{x \theta_{1}}, X_{x \theta_{2}}, X_{\theta_{1}}, X_{\theta_{2}}, X_{\theta_{1} \theta_{2}}\right) .
$$

Obviously, the Lie superalgebra $\mathfrak{o s p}(n-1 \mid 2)$ can be considered as a subalgebra of $\mathfrak{o s p}(n \mid 2)$, therefore, the spaces of symbols $\mathcal{S P}(n)$ are also osp $(n-1 \mid 2)$-modules.

We compute the cohomology spaces $\mathrm{H}^{1}(\mathfrak{o s p}(n \mid 2), \mathcal{S P}(n))$ and $\mathrm{H}^{1}(\mathfrak{o s p}(n \mid 2)$, $\mathcal{S} \Psi \mathcal{D O}(n)$ ), where $n=0,1,2$. We show that non vanishing spaces . To be more precise, let $d_{n}=\operatorname{dim}\left(\mathrm{H}_{\text {diff }}^{1}(\mathfrak{o s p}(n \mid 2), \mathcal{S P}(n))\right)=\operatorname{dim}\left(\mathrm{H}^{1}(\mathfrak{o s p}(n \mid 2), \mathcal{S} \Psi \mathcal{D O}(n))\right.$, we show that

$$
\begin{gathered}
d_{0}=d_{1}=2 \\
d_{2}=6 .
\end{gathered}
$$


These cohomology spaces are closely related to the deformation theory, see e.g. [4-6, 9, 10]. These spaces arise in the classification of infinitesimal deformations of the $\operatorname{osp}(n \mid 2)$-modules. We hope to be able to describe in the future all the deformations of these modules $\mathcal{S} \Psi \mathcal{D O}(n)$.

\section{$2 \mathfrak{o s p}(n \mid 2)-$ Modules}

$2.1 \mathfrak{s l}(2)$-module

Consider the space $\left.\mathbb{C}\left[\xi, \xi^{-1}\right]\right]$ of (formal) Laurent series of finite order in $\xi$ :

$$
a(\xi)=\sum_{-\infty}^{m} a_{k} \xi^{k}
$$

We put

$$
\left.\mathcal{P}:=\mathcal{C}^{\infty}(\mathbb{R}) \otimes \mathbb{C}\left[\xi, \xi^{-1}\right]\right],
$$

with its natural multiplication and the Poisson bracket defined as follows. Any element of $\mathcal{P}$ can be written in the following form:

$$
F(x, \xi)=\sum_{-\infty}^{m} f_{k}(x) \xi^{k},
$$

where $f_{k}(x) \in \mathcal{C}^{\infty}(\mathbb{R})$, then for $F, G \in \mathcal{P}$,

$$
\{F, G\}=\frac{\partial F}{\partial \xi} \frac{\partial G}{\partial x}-\frac{\partial F}{\partial x} \frac{\partial G}{\partial \xi} .
$$

The associative algebra of pseudodifferential symbols $\Psi \mathcal{D O}$ has the same underlying vector space, but the multiplication is now defined by the following formula

$$
F \circ G=\sum_{k \geq 0} \frac{1}{k !} \frac{\partial^{k} F}{\partial \xi^{k}} \frac{\partial^{k} G}{\partial x^{k}} .
$$

As usual, one can consider this associative algebra $\Psi D \mathcal{O}$ as a Lie algebra with the commutator

$$
[F, G]=F \circ G-G \circ F .
$$

The order of pseudodifferential operators defines a natural filtration on $\Psi D \mathcal{O}$. Recall, that the order is defined by $\operatorname{ord}(F)=\sup \left\{k \in \mathbb{Z} / f_{k}(x) \neq 0\right\}$, for every $F(x, \xi)=\sum_{k \in \mathbb{Z}} f_{k}(x) \xi^{k}$. One sets $\mathbf{F}_{n}=\{F \in \Psi D \mathcal{O} \mid \operatorname{ord}(F) \leq-n\}$, where $n \in \mathbb{Z}$. Thus, one has a decreasing filtration [10],

$$
\cdots \subset \mathbf{F}_{n+1} \subset \mathbf{F}_{n} \subset \cdots,
$$

compatible with multiplication, if $F \in \mathbf{F}_{n}$ and $G \in \mathbf{F}_{m}$, then $F \circ G \in \mathbf{F}_{n+m}$, and $\{F, G\} \in \mathbf{F}_{n+m-1}$ (check the symbolic terms!). This filtration makes $\Psi D \mathcal{O}$ an associative filtered algebra, one can consider, as usual, the associated graded algebra. Each quotient space $\mathbf{F}_{n} / \mathbf{F}_{n+1}$ is canonically isomorphic to $\mathcal{C}^{\infty}(\mathbb{R})$, any function 
$f \in \mathcal{C}^{\infty}(\mathbb{R})$ induces a pseudodifferential symbol $f \xi^{-n}$ which has a well-defined image in $\mathbf{F}_{n} / \mathbf{F}_{n+1}$. The associated graded algebra is then $\operatorname{Gr}(\Psi D \mathcal{O})=\widetilde{\bigoplus}_{n \in \mathbb{Z}} \mathbf{F}_{n} / \mathbf{F}_{n+1}$, where, $\bigoplus_{n \in \mathbb{Z}}=\left(\bigoplus_{n<0}\right) \oplus \prod_{n \geq 0}$

The defined filtration is also a filtration of $\Psi \mathcal{D O}$ as a $\mathfrak{v e c t}(1)$-module (i.e. compatible with the natural action of $\mathfrak{v e c t}(1)$ on $\Psi \mathcal{D O})$. Indeed, if $X \in \mathfrak{v e c t}(1)$ and $F \in \mathbf{F}_{n}(\Psi D \mathcal{O})$, then $X . F=[X, F] \in \mathbf{F}_{n}$. One induces an action of $\mathfrak{v e c t}(1)$ on the associative algebra $\operatorname{Gr}(\Psi D \mathcal{O})=\mathcal{P}$ and a simple computation shows that we recover the canonical action of $\mathfrak{v e c t}(1)$ on the Poisson algebra $\mathcal{P}$ [10]. More explicitly, as a $\mathfrak{v e c t}(1)$-module, $\mathbf{F}_{n} / \mathbf{F}_{n+1}=\mathcal{F}_{n}$, where $\mathcal{F}_{n}$ is the space of tensor densities of degree $\mathrm{n}$ on $\mathbb{R}$ :

$$
\mathcal{F}_{n}=\left\{f(x) d x^{n} \mid f \in \mathcal{C}^{\infty}(\mathbb{R})\right\}
$$

and the action of $\mathfrak{v e c t}(1)$ reads

$$
L_{X_{g}}^{n}\left(f d x^{n}\right)=\left(g f^{\prime}+n g^{\prime} f\right) d x^{n} .
$$

Note, that the expression in the right hand side is just the standard Lie derivative of a tensor density along a vector field. Here, we restrict ourselves to the subalgebra $\mathfrak{s l}(2)$, thus we obtain a $\mathfrak{s l}(2)$-modules still denoted by $\mathcal{P}$.

\section{$2.2 \mathfrak{o s p}(1 \mid 2)$-module}

The superspace of the supercommutative algebra of superpseudodifferential symbols on $\mathbb{R}^{1 \mid 1}$ with its natural multiplication is spanned by the series(see [1])

$$
\mathcal{S P}(1)=\left\{F=\sum_{k=-M}^{\infty} a_{k, \epsilon}\left(x, \theta_{1}\right) \xi^{-k} \bar{\theta}_{1}^{\epsilon}: a_{k, \epsilon} \in C^{\infty}\left(\mathbb{R}^{111}\right) ; \epsilon=0,1 ; M \in \mathbb{N}\right\},
$$

where $\xi$ corresponds to $\partial_{x}$ and $\overline{\theta_{1}}$ corresponds to $\partial_{\theta_{1}}\left(p\left(\overline{\theta_{1}}\right)=1\right)$.

The space $\mathcal{S P}(1)$ has a structure of the Poisson Lie superalgebra given by the following bracket (cf, [8]):

$$
\{F, G\}=\frac{\partial(F)}{\partial \xi} \frac{\partial(G)}{\partial x}-\frac{\partial(F)}{\partial x} \frac{\partial(G)}{\partial \xi}-(-1)^{p(F)}\left(\frac{\partial(F)}{\partial \theta_{1}} \frac{\partial(G)}{\partial \bar{\theta}_{1}}+\frac{\partial(F)}{\partial \bar{\theta}_{1}} \frac{\partial(G)}{\partial \theta_{1}}\right) .
$$

The space $\mathcal{S P}(1)$ has a structure of associative superalgebra defined by the following rule:

$$
F \circ G=\sum_{k \geq 0, v=0,1} \frac{(-1)^{p(F)+1}}{k !}\left(\partial_{\xi}^{k} \partial_{\bar{\theta}_{1}}^{\nu} F\right)\left(\partial_{x}^{k} \partial_{\theta_{1}}^{\nu} G\right) .
$$

This composition rule induces the supercommutator defined by:

$$
[F, G]=F \circ G-(-1)^{p(F) p(G)} G \circ F .
$$

The natural embedding of $\mathcal{K}(1)$ into $\mathcal{S P}(1)$ given by $\mathcal{K}(1)$ :

$$
\pi: X_{F} \longmapsto F \xi-\frac{(-1)^{p(F)}}{2} \bar{\eta}_{1}(F) \bar{\zeta}_{1} .
$$

where $\bar{\zeta}_{1}=\bar{\theta}_{1}-\theta_{1} \xi$, induces a $\mathcal{K}(1)$-module structure on it. 
Setting the degree of $x, \theta_{1}$ be zero and the degree of $\xi, \zeta_{1}$ be 1 we introduce a $\mathbb{Z}$-grading in the Poisson superalgebra $\mathcal{S P}(1)$ which will be simply denoted $\mathcal{S P}(1)$. Then we have

$$
\mathcal{S P}(1)=\widetilde{\bigoplus}_{n \in \mathbb{Z}} \mathcal{S} \mathcal{P}_{n}(1):=\left(\bigoplus_{n<0} \mathcal{S} \mathcal{P}_{n}(1)\right) \oplus\left(\prod_{n \geq 0} \mathcal{S} \mathcal{P}_{n}(1)\right),
$$

where $\mathcal{S} \mathcal{P}_{n}(1)=\left\{F \xi^{-n}+G \xi^{-n-1} \zeta_{1}: F, G \in C^{\infty}\left(\mathbb{R}^{1 \mid 1}\right)\right\}$ is the homogeneous subspace of degree $-n$.

Each element of $\mathcal{S} \Psi \mathcal{D O}(1)$ can be expressed as

$$
F=\sum_{k \in \mathbb{Z}}\left(F_{k}+G_{k} \eta_{1}^{-1}\right) \eta_{1}^{2 k}, \text { where } F_{k}, G_{k} \in C^{\infty}\left(\mathbb{R}^{1 \mid 1}\right)
$$

We define the order of $F$ to be

$$
\operatorname{ord}(F)=\sup \left\{k: F_{k}(x, \theta) \neq 0 \text { or } G_{k}(x, \theta) \neq 0\right\} .
$$

This definition of order equips $\mathcal{S} \Psi \mathcal{D O}$ (1) with a decreasing filtration as follows: set

$$
\mathbf{F}_{n}=\{F \in \mathcal{S P}(1): \operatorname{ord}(F) \leq-n\}, \text { where } n \in \mathbb{Z} .
$$

So one has (see [1])

$$
\ldots \subset \mathbf{F}_{n+1} \subset \mathbf{F}_{n} \subset \ldots
$$

This filtration is compatible with the multiplication and the super Poisson bracket, that is, for $F \in \mathbf{F}_{n}$ and $G \in \mathbf{F}_{m}$, one has $F \circ G \in \mathbf{F}_{n+m}$ and $\{F, G\} \in \mathbf{F}_{n+m-1}$, after as a vector spaces we identify $\mathcal{S P}(1)$ with $S \Psi \mathcal{D O}(1)$. This filtration makes $\mathcal{S P}(1)$ an associative filtered superalgebra. Moreover, this filtration is compatible with the natural action of $\mathcal{K}(1)$ on $\mathcal{S P}(1)$. Indeed, if $X_{F} \in \mathcal{K}(1)$ and $G \in \mathbf{F}_{n}$, then

$$
X_{F} \cdot G=\left[X_{F}, G\right] \in \mathbf{F}_{n} .
$$

The induced $\mathcal{K}(1)$-action on the quotient $\mathbf{F}_{n} / \mathbf{F}_{n+1}$ is isomorphic to the $\mathcal{K}(1)$-action on $\mathcal{S} \mathcal{P}_{n}$. Therefore, the $\mathcal{K}(1)$-action on the associated graded space of the filtration (2.24), is isomorphic to the graded $\mathcal{K}(1)$-module $\mathcal{S P}(1)$, that is (see [1])

$$
\mathcal{S P}(1) \simeq \widetilde{\bigoplus}_{n \in \mathbb{Z}} \mathbf{F}_{n} / \mathbf{F}_{n+1}
$$

If we restrict ourselves to the Lie subalgebra osp(1|2) of $\mathcal{K}(1)$, we get a family of infinite dimensional $\mathfrak{o s p}(1 \mid 2)$ modules, still denoted $\mathfrak{F}_{\lambda}^{1}$ and $\mathcal{S P}(1)$.

\section{$2.3 \mathfrak{o s p}(2 \mid 2)$-module}

Let $\mathcal{K}(2)$ be the Lie superagebra of vector fields on $\mathbb{R}^{1 / 2}$. The natural embedding of $\mathcal{K}(2)$ into $\mathcal{S P}(2)$ defined by

$$
\pi\left(X_{F}\right)=F \xi+\frac{(-1)^{p(F)+1}}{2} \sum_{i=1}^{2} \bar{\eta}_{i}(F) \bar{\zeta}_{i}, \text { where } \bar{\zeta}_{i}=\bar{\theta}_{i}-\theta_{i} \xi
$$

induces a $\mathcal{K}(2)$-module structure on $\mathcal{S P}(2)$. 
Setting $\operatorname{deg} x=\operatorname{deg} \theta_{i}=0, \operatorname{deg} \xi=\operatorname{deg} \bar{\theta}_{i}=1$ for all $i$, we endow the Poisson superalgebra $\mathcal{S P}(2)$ with a $\mathbb{Z}$-grading:

$$
\mathcal{S P}(2)=\widetilde{\bigoplus}_{n \in \mathbb{Z}} \mathcal{S P} \mathcal{P}_{n}(2)
$$

where $\widetilde{\bigoplus}_{n \in \mathbb{Z}}=\left(\bigoplus_{n<0}\right) \oplus \prod_{n \geq 0}$ and

$$
\mathcal{S} \mathcal{P}_{n}(2)=\left\{F \xi^{-n}+G \xi^{-n-1} \bar{\theta}_{1}+H \xi^{-n-1} \bar{\theta}_{2}+T \xi^{-n-2} \bar{\theta}_{1} \bar{\theta}_{2} \mid F, G, H, T \in C^{\infty}\left(\mathbb{R}^{1 \mid 2}\right)\right\}
$$

is the homogeneous subspace of degree $-n$. Each element of $\mathcal{S} \Psi \mathcal{D O}(2)$ can be expressed as

$$
A=\sum_{k \in \mathbb{Z}}\left(F_{k}+G_{k} \xi^{-1} \bar{\theta}_{1}+H_{k} \xi^{-1} \bar{\theta}_{2}+T_{k} \xi^{-2} \bar{\theta}_{1} \bar{\theta}_{2}\right) \xi^{-n}
$$

where $F_{k}, G_{k}, H_{k}, T_{k} \in C^{\infty}\left(\mathbb{R}^{1 / 2}\right)$. We define the order of $A$ to be

$$
\begin{aligned}
\operatorname{ord}(A) & =\sup \left\{k \mid F_{k}\left(x, \theta_{1}, \theta_{2}\right) \neq 0 \text { or } G_{k}\left(x, \theta_{1}, \theta_{2}\right) \neq 0 \text { or } H_{k}\left(x, \theta_{1}, \theta_{2}\right)\right. \\
& \left.\neq 0 \text { or } T_{k}\left(x, \theta_{1}, \theta_{2}\right) \neq 0\right\}
\end{aligned}
$$

This definition of order equips $\mathcal{S} \Psi \mathcal{D O}$ (2) with a decreasing filtration as follows: set

$$
\mathbf{F}_{n}=\{A \in \mathcal{S} \Psi \mathcal{D O O}(2), \operatorname{ord}(A) \leq-n\},
$$

where $n \in \mathbb{Z}$. So one has

$$
\ldots \subset \mathbf{F}_{n+1} \subset \mathbf{F}_{n} \subset \ldots
$$

This filtration is compatible with the multiplication and the Poisson bracket, that is, for $A \in \mathbf{F}_{n}$ and $B \in \mathbf{F}_{m}$, one has $A \circ B \in \mathbf{F}_{n+m}$ and $\{A, B\} \in \mathbf{F}_{n+m-1}$. This filtration makes $\mathcal{S} \Psi \mathcal{D O}(2)$ an associative filtered superalgebra. Consider the associated graded space

$$
\operatorname{Gr}(\mathcal{S} \Psi \mathcal{D O}(2))=\widetilde{\bigoplus}_{n \in \mathbb{Z}} \mathbf{F}_{n} / \mathbf{F}_{n+1}
$$

The filtration (2.24) is also compatible with the natural action of $\mathcal{K}(2)$ on $\mathcal{S} \Psi \mathcal{D O}(2)$. Indeed, if $X_{F} \in \mathcal{K}(2)$ and $A \in \mathbf{F}_{n}$, then

$$
X_{F} \cdot A=\left[X_{F}, A\right] \in \mathbf{F}_{n} .
$$

The induced $\mathcal{K}(2)$-module on the quotient $\mathbf{F}_{n} / \mathbf{F}_{n+1}$ is isomorphic to the $\mathcal{K}(2)$-module $\mathcal{S P}_{n}(2)$. Therefore, the $\mathcal{K}(2)$-module $\operatorname{Gr}(\mathcal{S} \Psi \mathcal{D O O}(2))$, is isomorphic to the graded $\mathcal{K}(2)$-module $\mathcal{S P}(2)$, that is

$$
\mathcal{S P}(2) \simeq \widetilde{\bigoplus_{n \in \mathbb{Z}}} \mathbf{F}_{n} / \mathbf{F}_{n+1}
$$

If we restrict ourselves to the Lie subalgebra $\mathfrak{o s p}(2 \mid 2)$ of $\mathcal{K}(2)$, we get a family of infinite dimensional $\mathfrak{o s p}(2 \mid 2)$ modules, still denoted $\mathfrak{F}_{\lambda}^{2}$ and $\mathcal{S P}(2)$. 
Recall that a $C^{\infty}$ function on $\mathbb{R}^{1 \mid 2}$ has the form $F=f_{0}+f_{1} \theta_{1}+f_{2} \theta_{2}+f_{12} \theta_{1} \theta_{2}$ with $f_{0}, f_{1}, f_{2}, f_{12} \in C^{\infty}(\mathbb{R})$ and a $C^{\infty}$ function on $\mathbb{R}_{i}^{1 \mid 1}(i=1,2)$, where $\mathbb{R}_{i}^{1 \mid 1}$ is the superligne with local coordinates $\left(x, \theta_{i}\right)$, has the form $F=f_{0}+f_{i} \theta_{i}\left(f_{12}=f_{3-i}=0\right)$ with $f_{0}, f_{i} \in C^{\infty}(\mathbb{R})$. Then the Lie superalgebra $\mathfrak{o s p}(2 \mid 2)$ has two subsuperalgebras $\mathfrak{o s p}(1 \mid 2)_{i}$ for $i=1,2$ isomorphic to $\mathfrak{o s p}(1 \mid 2)$ defined by

$$
\mathfrak{o s p}(1 \mid 2)_{i}=\operatorname{Span}\left(X_{1}, X_{x}, X_{x^{2}}, X_{x \theta_{i}}, X_{\theta_{i}}\right) .
$$

Therefore, $\mathcal{S P}(2)$ and $\mathfrak{F}_{\lambda}^{i}$ are $\mathfrak{o s p}(1 \mid 2)_{i}$-modules.

For $i=1,2$, let $\mathfrak{F}_{\lambda}^{1}$ be the $\mathfrak{o s p}(1 \mid 2)_{i}$-module of weighted densities of weight $\lambda$ on $\mathbb{R}_{i}^{1 \mid 1}$.

\section{Cohomology}

Let $\mathfrak{g}=\mathfrak{g}_{0} \oplus \mathfrak{g}_{\overline{1}}$ be a Lie superalgebra acting on a superspace $V=V_{\overline{0}} \oplus V_{\overline{1}}$. The space of $k$-cochains of $\mathfrak{g}$ with values in $V$ is the $\mathfrak{g}$-module

$$
C^{k}(\mathfrak{g}, V):=\operatorname{Hom}\left(\Lambda^{k} \mathfrak{g} ; V\right) .
$$

The coboundary operator $\delta_{k}: C^{k}(\mathfrak{g}, V) \longrightarrow C^{k+1}(\mathfrak{g}, V)$ is a $\mathfrak{g}$-map satisfying $\delta_{n} \circ$ $\delta_{k-1}=0$. The kernel of $\delta_{k}$, denoted $Z^{k}(\mathfrak{g}, V)$, is the space of $k$-cocycles, among them, the elements in the range of $\delta_{k-1}$ are called $k$-coboundaries. We denote $B^{k}(\mathfrak{g}, V)$ the space of $k$-coboundaries.

By definition, the $k^{\text {th }}$ cohomology space is the quotient space

$$
\mathrm{H}^{k}(\mathfrak{g}, V)=Z^{k}(\mathfrak{g}, V) / B^{k}(\mathfrak{g}, V) .
$$

We will only need the formula of $\delta_{n}$ (which will be simply denoted $\delta$ ) in degrees 0,1 and 2: for $v \in C^{0}(\mathfrak{g}, V)=V, \delta v(x):=(-1)^{p(x) p(v)} x \cdot v$, for $\Upsilon \in C^{1}(\mathfrak{g}, V)$,

$$
\delta(\Upsilon)(x, y):=(-1)^{p(x) p(\Upsilon)} x \cdot \Upsilon(y)-(-1)^{p(y)(p(x)+p(\Upsilon))} y \cdot \Upsilon(x)-\Upsilon([x, y])
$$

In this paper, we study the differential cohomology spaces $\mathrm{H}^{1}(\mathfrak{o s p}(n \mid 2), \mathcal{S P}(n))$ and $\mathrm{H}^{1}(\mathfrak{o s p}(n \mid 2), \mathcal{S} \Psi \mathcal{D O}(n))$, where $n=0,1,2$.

\subsection{The Spectral Sequence for a Filtered Module Over a Lie (super)algebra}

Let $\mathfrak{g}$ be a Lie (super)algebra and $M$ a filltered module with decreasing filtration $\left\{M_{n}\right\}_{n \in \mathbb{Z}}$ so that $M_{n+1} \subset M_{n}, \cup_{n \in \mathbb{Z}} M_{n}=M$ and $\mathfrak{g} . M_{n} \subset M_{n}$. Let

$$
\operatorname{Gr}(M)=\widetilde{\bigoplus}_{n \in \mathbb{Z}} M_{n} / M_{n+1}
$$

be the associated graded $\mathfrak{g}$-module and $G r^{n}(M)=M_{n} / M_{n+1}$. One can then naturally construct a filtration on the space of cochains by setting $F_{n}\left(C^{*}(\mathfrak{g} ; M)\right)=C^{*}\left(\mathfrak{g} ; M_{n}\right)$, this filtration being obviously compatible with the Chevalley-Eilenberg differential. Then we have: $d F_{n}\left(C^{*}(\mathfrak{g}, M)\right) \subset F_{n}\left(C^{*}(\mathfrak{g}, M)\right)$ (i.e., the filtration is preserved by d); $F_{n+1}\left(C^{*}(\mathfrak{g}, M)\right) \subset F_{n}\left(C^{*}(\mathfrak{g}, M)\right)$ (i.e. the filtration is decreasing). and

Hence there is a spectral sequence $\left(E_{k}^{*, *}, d_{k}\right)$ for $k \in \mathbb{N}$ with dr of degree $(k, 1-k)$

$$
E_{0}^{p, q}=F_{p}\left(C^{p+q}(\mathfrak{g} ; M)\right) / F_{p-1}\left(C^{p+q}(\mathfrak{g} ; M)\right)
$$


and

$$
E_{1}^{p, q}=H^{p+q}\left(\mathfrak{g} ; G r^{p}(M)\right)
$$

where $G r^{p}(M)$

We define

$$
\begin{gathered}
Z_{k}^{p, q}=F_{p} C^{p+q} \bigcap d^{-1}\left(F_{p+k} C^{p+q+1}\right), \\
B_{k}^{p, q}=F_{p} C^{p+q} \bigcap d\left(F_{p-k} C^{p+q-1}\right), \\
E_{k}^{p, q}=Z_{k}^{p, q} /\left(Z_{k-1}^{p+1, q-1}+B_{k-1}^{p, q}\right) .
\end{gathered}
$$

The differential $d$ maps $Z_{k}^{p, q}$ into $Z_{k}^{p+k, q-k+1}$, and hence includes a homomorphism

$$
d_{k}: E_{k}^{p, q} \longrightarrow E_{k}^{p+k, q-k+1}
$$

The spectral sequence converges to $H^{*}(C, d)$, that is

$$
E_{\infty}^{p, q} \simeq F_{p} H^{p+q}(C, d) / F_{p+1} H^{p+q}(C, d),
$$

where $F_{p} H^{*}(C, d)$ is the image of the map $H^{*}\left(F_{p} C, d\right) \rightarrow H^{*}(C, d)$ induced by the inclusion $F_{p} C \rightarrow C$ (see $\left.[2,10]\right)$.

\subsection{Cohomology of $\mathfrak{s l}(2)$}

We compute the space $\mathrm{H}^{1}(\mathfrak{s l}(2), \mathcal{P})$. The result is the following:

\section{Theorem 3.1}

$$
\mathrm{H}^{1}(\mathfrak{s l}(2), \mathcal{P}) \simeq \mathbb{R}^{2}
$$

The nontrivial spaces $\left.\mathrm{H}^{1}(\mathfrak{s l}(2), \mathcal{P})\right)$ are spanned by the cohomology classes of the 1cocycles $\chi_{n}$ defined by:

$$
\chi_{0}\left(X_{f}\right)=f^{\prime} \text { and } \chi_{1}\left(X_{f}\right)=f^{\prime \prime} \xi^{-1} .
$$

In our case we can use the results in [3]. One has:

$$
\mathrm{H}^{1}\left(\mathfrak{s l}(2), \mathcal{F}_{\lambda}\right) \simeq\left\{\begin{array}{l}
\mathbb{R} \text { if } \lambda=0,1 \\
0 \text { otherwise }
\end{array}\right.
$$

The nontrivial spaces $\left.\mathrm{H}^{1}\left(\mathfrak{s l}(2), \mathcal{F}_{\lambda}\right)\right)$ are spanned by the cohomology classes of the 1-cocycles $\beta_{\lambda}$ defined by:

$$
\beta_{0}\left(X_{f}\right)=f^{\prime} \quad \text { and } \quad \beta_{1}\left(X_{f}\right)=f^{\prime \prime} d x .
$$

Proposition 3.2 [10] As a $\mathfrak{s l ( 2 ) - m o d u l e ~ w e ~ h a v e ~}$

$$
\mathcal{P} \simeq \widetilde{\bigoplus}_{n \in \mathbb{Z}} \mathcal{F}_{n}
$$

To prove Proposition 3.2, we need the following result (see [10]). 
Proof According to Proposition 3.2 and using the cohomology space (3.27), we obtain that the space of $\mathfrak{s l}(2)$ with coefficients in the space of symbols $\mathcal{P}$ has the following structure

$$
\mathrm{H}^{1}(\mathfrak{s l}(2), \mathcal{P})=\widetilde{\bigoplus}_{n \in \mathbb{Z}} \mathrm{H}^{1}\left(\mathfrak{s l}(2), \mathcal{F}_{n}\right)=\mathbb{R}^{2}
$$

It is generated by the non-trivial cohomology classes of $\chi_{0}\left(X_{f}\right)=f^{\prime}$ and $\chi_{1}\left(X_{f}\right)=$ $f^{\prime \prime} \xi^{-1}$ corresponding $\beta_{0}$ and $\beta_{1}$ respectively.

\section{Corollary 3.1}

$$
\mathrm{H}^{1}(\mathfrak{s l}(2), \Psi \mathcal{D O}) \simeq \mathbb{R}^{2} .
$$

The nontrivial spaces $\mathrm{H}^{1}(\mathfrak{s l}(2), \Psi \mathcal{D O})$ are spanned by the cohomology classes of the 1-cocycles $\Xi_{n}$ defined by:

$$
\Xi_{0}\left(X_{f}\right)=f^{\prime} \quad \text { and } \quad \Xi_{1}\left(X_{f}\right)=f^{\prime \prime} \xi^{-1} .
$$

Proof The filtration (2.24) is compatible with the multiplication and the Poisson bracket, that is, for $F \in \mathbf{F}_{n}$ and $G \in \mathbf{F}_{m}$, one has $F \circ G \in \mathbf{F}_{n+m}$ and $\{F, G\} \in \mathbf{F}_{n+m-1}$, after we identify $\mathcal{P}$ with $\Psi \mathcal{D O}$. Then $\mathrm{H}^{1}(\mathfrak{s l}(2), \Psi \mathcal{D O})=\mathrm{H}^{1}(\mathfrak{s l}(2), \mathcal{P})$. Now, as Ovsienko and Roger, we will use the constructed spectral sequence to compute the explicit expressions of the basis cocycles.

Indeed, the differential $d_{1}$ is

$$
d_{1}: E_{1}^{p, q} \rightarrow E_{1}^{p+1, q} .
$$

One can check it in the following way: consider a cocycle with values in $\mathcal{P}$, but compute its boundary as if it was with values in $\Psi \mathcal{D O}$ and keep the symbolic part of the result. This gives a new cocycle of degree equal to the degree of the previous one plus one, and its class will represent its image by $d_{1}$. The higher order differentials

$$
d_{k}: E_{k}^{p, q} \rightarrow E_{k}^{p+k, q-k+1}
$$

can be constructed by iteration of this procedure, the space $E_{k}^{p+k, q-k+1}$ contains the subspace coming from $H^{p+q+1}\left(\mathfrak{s l}(2) ; G r^{p+1}(\Psi \mathcal{D O})\right)$, where $G r^{p}(\Psi \mathcal{D O})$ is isomorphic, as $\mathfrak{s l}(2)$-module to the space of weighted densities $\mathcal{F}_{p}$.

It is easy to see that the cocycles $\chi_{0}$ and $\chi_{1}$ will survive in the same form, we will denote them $\Xi_{0}$ and $\Xi_{1}$ when seen as cocycles with values in $\Psi \mathcal{D O}$.

\subsection{Cohomology of $\mathfrak{o s p}(1 \mid 2)$}

The main result in this subsection is the following:

Theorem 3.3

$$
\mathrm{H}^{1}(\mathfrak{o s p}(1 \mid 2), \mathcal{S P}(1)) \simeq \mathbb{R}^{2}
$$

The nontrivial spaces $\mathrm{H}^{1}(\mathfrak{o s p}(1 \mid 2), \mathcal{S P}(1))$ are spanned by the following 1-cocycles:

$$
\varepsilon_{0}\left(X_{F}\right)=F^{\prime} \quad \text { and } \quad \varepsilon_{1}\left(X_{F}\right)=\eta_{1}^{3}(F) \xi^{-1} \zeta_{1}-2 \theta_{1} \eta_{1}^{3}(F)
$$




\section{Proposition $3.4[3]$}

$$
\mathrm{H}^{1}\left(\mathfrak{o s p}(1 \mid 2), \mathfrak{F}_{\lambda}^{1}\right) \simeq\left\{\begin{array}{l}
\mathbb{R} \text { if } \lambda=0, \frac{1}{2} \\
0 \text { otherwise. }
\end{array}\right.
$$

The nontrivial spaces $\mathrm{H}^{1}\left(\mathfrak{o s p}(1 \mid 2), \mathfrak{F}_{\lambda}^{1}\right)$ are spanned by the following 1-cocycles:

$$
\epsilon_{0}\left(X_{F}\right)=F^{\prime} \quad \text { and } \quad \epsilon_{\frac{1}{2}}\left(X_{F}\right)=\eta_{1}^{3}(F) \alpha^{\frac{1}{2}} .
$$

To prove Proposition 3.4, we need the following result (see [3]).

Proposition 3.5 [1] As a osp(1|2)-module we have

$$
\mathcal{S P}(1) \simeq \widetilde{\bigoplus}_{n \in \mathbb{Z}}\left(\mathfrak{F}_{n}^{1} \oplus \mathfrak{F}^{1}{ }_{n+\frac{1}{2}}\right) .
$$

To prove Proposition 3.5, we need the following result (see [1]).

Proof According to Proposition 3.4 and Eq. 2.23, we obtain that the space of $\mathfrak{o s p}(1 \mid 2)$ with coefficients in the space of symbols $\mathcal{S P}_{n}$ has the following structure

$$
\mathrm{H}^{1}\left(\mathfrak{o s p}(1 \mid 2), \mathcal{S} \mathcal{P}_{n}(1)\right) \simeq \begin{cases}\mathbb{R}^{2} & \text { if } n=0 \\ 0 & \text { otherwise }\end{cases}
$$

Using to Proposition 3.5, we obtain that the space of $\mathfrak{o s p}(1 \mid 2)$ with coefficients in the space of symbols $\mathcal{S P}(1)$ has the following structure

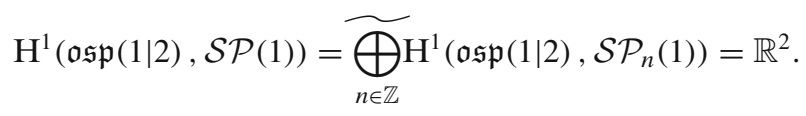

It is generated by the non-trivial cohomology classes of $\bar{\varepsilon}_{0}=\varepsilon_{0}+\varepsilon_{1}$ and $\varepsilon_{1}$ corresponding $\epsilon_{0}$ and $\epsilon_{\frac{1}{2}}$ respectively.

\section{Corollary 3.2}

$$
\mathrm{H}^{1}(\mathfrak{o s p}(1 \mid 2), \mathcal{S} \Psi \mathcal{D O}(1)) \simeq \mathbb{R}^{2} .
$$

The nontrivial spaces $\mathrm{H}^{1}(\mathfrak{o s p}(1 \mid 2), \mathcal{S} \Psi \mathcal{D O}(1))$ are spanned by the cohomology classes of the 1-cocycles $\Lambda_{n}$ defined by:

$$
\Lambda_{0}\left(X_{F}\right)=F^{\prime} \quad \text { and } \quad \Lambda_{1}\left(X_{F}\right)=\eta_{1}^{3}(F) \bar{\eta}_{1}^{-3}+\bar{\eta}^{4}(F) \bar{\eta}_{1}^{-2} .
$$

Proof The cohomology space $\mathrm{H}^{1}(\mathfrak{o s p}(1 \mid 2), \mathcal{S} \Psi \mathcal{D O}(1))$ is obviously upper-bounded by $\mathrm{H}^{1}(\mathfrak{o s p}(1 \mid 2), \mathcal{S P}(1))$, we have to find explicit expressions for the non trivial cocycles generating the former cohomology. To constrict these cocycles, we follow Ovsienko and Roger in [10] based on the computations of successive differentials of the spectral sequences corresponding to the complex $C^{*}(\mathfrak{o s p}(1 \mid 2), \mathcal{S P}(1))$ to compute the explicit expressions of the basis cocycles: consider a cocycle with values in $\mathcal{S P}$, but compute its boundary as if it was with values in $\mathcal{S} \Psi \mathcal{D O}$ (1) and keep the symbolic part of the result. This gives a new cocycle of degree equal to the degree of the previous one plus one, and its class will represent its image by $d_{1}$. It 
is generated by the non-trivial cohomology classes of $\Lambda_{0}\left(X_{F}\right)=F^{\prime}$ and $\Lambda_{1}\left(X_{F}\right)=$ $\eta_{1}^{3}(F) \bar{\eta}_{1}^{-3}+\bar{\eta}^{4}(F) \bar{\eta}_{1}^{-2}$ corresponding $\varepsilon_{0}$ and $\varepsilon_{1}$ respectively.

3.4 Cohomology of $\mathfrak{o s p}(2 \mid 2)$

$\operatorname{As} \mathfrak{o s p}(1 \mid 2)_{i}$-isomorphism:

$$
\mathfrak{o s p}(2 \mid 2) \simeq \mathfrak{o s p}(1 \mid 2)_{i} \oplus \Pi\left(\mathcal{H}_{i}\right),
$$

where $\mathcal{H}_{i}$ is the subspace of $\mathfrak{F}_{-\frac{1}{2}}^{1}$ spanned by $\left\{\theta_{i} \alpha_{1}^{-\frac{1}{2}}, x \alpha_{1}^{-\frac{1}{2}}, \alpha_{1}^{-\frac{1}{2}}\right\}$ where $i=1,2$. To be more precise, any element $X_{F}$ is decomposed into $X_{F}=X_{F_{i}}+X_{F_{3-i} \theta_{3-i}}$ where $\partial_{3-i} F_{i}=\partial_{3-i} F_{3-i}=0$, and then $X_{F_{i}} \in \mathfrak{o s p}(1 \mid 2)_{i}$ and $X_{F_{3-i} \theta_{3-i}}$ can be identified to $\Pi\left(F_{3-i} \alpha_{1}^{-\frac{1}{2}}\right) \in \Pi\left(\mathcal{H}_{i}\right)$. Moreover, we can see easily that

$$
\left[\mathfrak{o s p}(1 \mid 2)_{i}, \Pi\left(\mathcal{H}_{i}\right)\right] \subset \Pi\left(\mathcal{H}_{i}\right) \quad \text { and } \quad\left[\Pi\left(\mathcal{H}_{i}\right), \Pi\left(\mathcal{H}_{i}\right)\right] \subset \mathfrak{o s p}(1 \mid 2)_{i} .
$$

The first cohomology space $\mathrm{H}^{1}\left(\mathfrak{o s p}(1 \mid 2)_{i}, \mathfrak{F}_{\lambda}^{2}\right)$ was computed in [3]. The result is the following:

$$
\mathrm{H}^{1}\left(\mathfrak{o s p}(1 \mid 2)_{i}, \mathfrak{F}_{\lambda}^{2}\right) \simeq \begin{cases}\mathbb{R}^{2} & \text { if } \lambda=0, \\ \mathbb{R} & \text { if } \lambda=-\frac{1}{2}, \frac{1}{2} \\ 0 & \text { otherwise }\end{cases}
$$

The respective nontrivial 1-cocycles are

$$
\begin{array}{ll}
C_{1}\left(X_{F_{i}}\right)=F_{i}^{\prime}, C_{2}\left(X_{F}\right)=\eta_{i}\left(F_{i}^{\prime}\right) \theta_{3-i} & \text { if } \lambda=0, \\
C_{3}\left(X_{F_{i}}\right)=F_{i}^{\prime} \theta_{3-i} \alpha_{2}^{-\frac{1}{2}} & \text { if } \lambda=-\frac{1}{2}, \\
C_{4}\left(X_{F_{i}}\right)=\eta_{i}^{3}\left(F_{i}\right) \alpha_{2}^{\frac{1}{2}} & \text { if } \lambda=\frac{1}{2},
\end{array}
$$

where $X_{F_{i}} \in \mathfrak{o s p}(1 \mid 2)_{i}$.

\section{Proposition 3.6 [2]}

1) As a osp $(1 \mid 2)_{i}$-module, $i=1$, 2, we have

$$
\mathcal{S P} \mathcal{P}_{n}(2) \simeq \mathfrak{F}_{n}^{2} \oplus \Pi\left(\mathfrak{F}_{n+\frac{1}{2}}^{2} \oplus \mathfrak{F}_{n+\frac{1}{2}}^{2}\right) \oplus \mathfrak{F}_{n+1}^{2} \text { for } n=0,-1
$$

2) For $n \neq 0,-1$ :

a) The following subspace of $\mathcal{S P}_{n}(2)$ :

$$
\mathcal{S P}_{n, i}(2)=\left\{\begin{array}{c}
B_{F}^{(n, i)}=F \theta_{3-i} \bar{\theta}_{3-i} \xi^{-n-1}+\theta_{3-i}\left(\bar{\eta}_{3-i}-\frac{1}{2} \bar{\eta}_{i}\right)(F) \bar{\zeta}_{i} \bar{\zeta}_{3-i} \xi^{-n-2} \mid \\
F \in C^{\infty}\left(\mathbb{R}^{1 \mid 2}\right)
\end{array}\right\}
$$

is a osp $(1 \mid 2)_{i}$ - module, $i=1,2$, isomorphic to $\mathfrak{F}_{n+1}^{2}$.

b) As a osp $(1 \mid 2)_{i}$-module we have

$$
\mathcal{S P}_{n}(2) / \mathcal{S} \mathcal{P}_{n, i}(2) \simeq \mathfrak{F}_{n}^{2} \oplus \Pi\left(\mathfrak{F}_{n+\frac{1}{2}}^{2} \oplus \mathfrak{F}_{n+\frac{1}{2}}^{2}\right), \quad i=1,2
$$


To prove Proposition 3.6, we need the following result (see [2]).

The space $\mathrm{H}^{1}(\mathfrak{o s p}(2 \mid 2), \mathcal{S P}(2))$ inherits the grading (2.23) of $\mathcal{S P}(2)$, so it suffices to compute it in each degree. The main result in this paper is the following:

\section{Theorem 3.7}

$$
\mathrm{H}^{1}(\mathfrak{o s p}(2 \mid 2), \mathcal{S P}(2)) \simeq \mathbb{R}^{6}
$$

The nontrivial spaces $\mathrm{H}^{1}(\mathfrak{o s p}(2 \mid 2), \mathcal{S P}(2))$ are spanned by the following 1-cocycles:

$$
\begin{aligned}
& \Upsilon_{1}\left(X_{F}\right)=(-1)^{p(F)} \eta_{1} \eta_{2}(F) \xi^{-1} \bar{\zeta}_{1} \bar{\zeta}_{2} \\
& \Upsilon_{2}\left(X_{F}\right)=F^{\prime} \xi^{-1} \bar{\zeta}_{1} \bar{\zeta}_{2} \\
& \Upsilon_{3}\left(X_{F}\right)=F^{\prime} \\
& \Upsilon_{4}\left(X_{F}\right)=(-1)^{p(F)} \eta_{1} \eta_{2}(F) \\
& \Upsilon_{5}\left(X_{F}\right)=(-1)^{p(F)}\left(\bar{\eta}_{1}^{3}(F) \bar{\zeta}_{1}+\bar{\eta}_{2}^{3}(F) \bar{\zeta}_{2}\right) \xi^{-1} \\
& \Upsilon_{6}\left(X_{F}\right)=F^{\prime \prime} \xi^{-2} \bar{\zeta}_{1} \bar{\zeta}_{2}+(-1)^{p(F)}\left(\bar{\eta}_{1}^{3}(F) \bar{\zeta}_{2}-\bar{\eta}_{2}^{3}(F) \bar{\zeta}_{1}\right) \xi^{-1}
\end{aligned}
$$

We know that any element $\Upsilon \in Z^{1}\left(\mathfrak{o s p}(2 \mid 2), \mathcal{S P}_{n}(2)\right)$ is decomposed into $\Upsilon=$ $\Upsilon^{\prime}+\Upsilon^{\prime \prime}$ where $\Upsilon^{\prime} \in Z^{1}\left(\mathfrak{o s p}(1 \mid 2)_{i}, \mathcal{S} \mathcal{P}_{n}(2)\right)$ and $\Upsilon^{\prime \prime} \in \operatorname{Hom}\left(\Pi\left(\mathcal{H}_{i}\right), \mathcal{S} \mathcal{P}_{n}(2)\right)$.

Proof To prove Theorem 3.7, we need first to prove the following lemma:

Lemma 3.8 The 1-cocycle $\Upsilon \in \mathbb{Z}^{1}\left(\mathfrak{o s p}(2 \mid 2), \mathcal{S} \mathcal{P}_{n}(2)\right), n \in \mathbb{Z}$ is a coboundary if and only if $\Upsilon^{\prime}$ is a coboundary.

Proof It is easy to see that if $\Upsilon$ is a coboundary for $\mathfrak{o s p}(2 \mid 2)$ then $\Upsilon^{\prime}$ is a coboundary over $\mathfrak{o s p}(1 \mid 2)_{i}$. Now assume that $\Upsilon^{\prime}$ is a coboundary over $\mathfrak{o s p}(1 \mid 2)_{i}$, that is, there exists $A \in \mathcal{S P}_{n}(2)$ such that for all $X_{F_{i}}$

$$
\Upsilon\left(X_{F_{i}}\right)=\left\{X_{F_{i}}, A\right\} .
$$

Using the condition of a 1-cocycle, we prove that

$$
\Upsilon\left(X_{\theta_{1} \theta_{2}}\right)=\left\{X_{\theta_{1} \theta_{2}}, A\right\} .
$$

We deduce that $\Upsilon\left(X_{F}\right)=\left\{X_{F}, A\right\}$, for any $X_{F} \in \mathfrak{o s p}(2 \mid 2)$, and therefore $\Upsilon$ is a coboundary of $\mathfrak{o s p}(2 \mid 2)$.

Proof of Theorem 3.7 First, according to Lemma 3.8, the restriction of any nontrivial 1 -cocycle of $\mathfrak{o s p}(2 \mid 2)$ with coefficients in $\mathcal{S P}_{n}(2)$ to $\mathfrak{o s p}(1 \mid 2)_{1}$ or to $\mathfrak{o s p}(1 \mid 2)_{2}$ is a nontrivial 1-cocycle.

As in [2], using Propositions 3.6 and 3.4, we obtain:

$$
\mathrm{H}^{1}\left(\mathfrak{o s p}(1 \mid 2)_{i}, \mathcal{S P}_{n}(2)\right) \simeq \begin{cases}\mathbb{R}^{4} \text { if } n=-1 \\ \mathbb{R}^{4} \text { if } n=0 \\ 0 \quad \text { otherwise }\end{cases}
$$

So, we see that if $n \neq 0,-1$, then by Lemma 3.8, the corresponding cohomology $\mathrm{H}^{1}\left(\mathfrak{o s p}(2 \mid 2), \mathcal{S} \mathcal{P}_{n}(2)\right)$ vanishes. 
Case $1 \quad n=-1$, the space $\mathrm{H}^{1}\left(\mathfrak{o s p}(1 \mid 2)_{i}, \mathcal{S} \mathcal{P}_{-1}(2)\right)$ is spanned by the following 1 cocyles:

$$
\begin{gathered}
\Phi_{1}^{i}\left(X_{F}\right)=F_{i}^{\prime} \theta_{3-i} \bar{\theta}_{3-i}+\theta_{3-i}\left(\bar{\eta}_{3-i}-\frac{1}{2} \bar{\eta}_{i}\right)\left(F_{i}^{\prime}\right) \bar{\zeta}_{i} \bar{\zeta}_{3-i} \xi^{-1}, \\
\Phi_{2}^{i}\left(X_{F}\right)=\theta_{3-i}\left(\bar{\eta}_{3-i}-\frac{1}{2} \bar{\eta}_{i}\right)\left(\eta_{i}\left(F_{i}^{\prime}\right) \theta_{3-i}\right) \bar{\zeta}_{i} \bar{\zeta}_{3-i} \xi^{-1}, \\
\Phi_{3}^{i}\left(X_{F}\right)=F_{i}^{\prime} \bar{\zeta}_{i}-\left(\theta_{3-i} \bar{\eta}_{i}+\theta_{i} \partial_{\theta_{3-i}}\right)\left(F_{i}^{\prime}\right) \bar{\theta}_{3-i}-(-1)^{p\left(F_{i}\right)} \partial_{\theta_{3-i}}\left(F_{i}^{\prime}\right) \bar{\theta}_{i} \bar{\theta}_{3-i} \xi^{-1}, \\
\widetilde{\Phi}_{3}^{i}\left(v_{F}\right)=F_{i}^{\prime} \bar{\zeta}_{i}+\left(1-\theta_{3-i} \bar{\eta}_{i}\right)\left(F_{i}^{\prime}\right) \bar{\theta}_{3-i} .
\end{gathered}
$$

Case $2 n=0$, the space $\mathrm{H}^{1}\left(\mathfrak{o s p}(1 \mid 2)_{i}, \mathcal{S P}_{0}(2)\right)$ is spanned by the following 1-cocyle:

$$
\begin{gathered}
\Phi_{4}^{i}\left(X_{F}\right)=F_{i}^{\prime}, \\
\Phi_{5}^{i}\left(X_{F}\right)=\eta_{i}\left(F^{\prime}\right) \theta_{3-i}, \\
\Phi_{6}^{i}\left(X_{F}\right)=\theta_{i} \Pi\left(C_{4}\left(X_{F}\right)\right)-\left(1-2 \theta_{3-i} \partial_{\theta_{3-i}}\right)\left(\Pi\left(C_{4}\left(X_{F}\right)\right)\right) \bar{\theta}_{i} \xi^{-1} \\
-\theta_{3-i} \partial_{\theta_{i}}\left(\Pi\left(C_{4}\left(X_{F}\right)\right)\right) \bar{\theta}_{3-i} \xi^{-1}+\left(\Pi\left(C_{4}\left(X_{F}\right)\right)\right)^{\prime} \theta_{3-i} \bar{\theta}_{i} \bar{\theta}_{3-i} \xi^{-2}, \\
\widetilde{\Phi}_{6}^{i}\left(X_{F}\right)=\theta_{i}\left(\partial_{\theta_{3-i}}-2 \partial_{\theta_{i}}+2 \theta_{3-i} \partial_{\theta_{3-i}} \partial_{\theta_{i}}\right)\left(\Pi\left(C_{4}\left(X_{F}\right)\right)\right) \bar{\theta}_{3-i} \xi^{-1} \\
+\frac{1}{2}\left(3-(-1)^{p(F)}\right)\left(\Pi\left(C_{4}\left(X_{F}\right)\right)\right) \bar{\theta}_{3-i} \xi^{-1} \\
+(-1)^{p\left(\Pi\left(C_{4}\left(X_{F}\right)\right)\right)}\left(\partial_{\theta_{3-i}}-\partial_{\theta_{i}}+\theta_{i} \partial_{x}\right)\left(\Pi\left(C_{4}\left(X_{F}\right)\right)\right) \bar{\theta}_{i} \bar{\theta}_{3-i} \xi^{-2}\left(\Pi\left(C_{4}\left(X_{F}\right)\right)\right),
\end{gathered}
$$

where the cocycle $C_{4}$ is defined by the formulae (3.33).

Now, consider a nontrivial 1-cocycle of $\mathfrak{o s p}(2 \mid 2)$ with coefficients in $\mathcal{S} \mathcal{P}_{n}(2)$ can be decomposed as $\Upsilon=\left(\Upsilon^{\prime}, \Upsilon^{\prime \prime}\right)$ and

$$
\left\{\begin{array}{l}
\Upsilon^{\prime}: \mathfrak{o s p}(1 \mid 2)_{i} \longrightarrow \mathcal{S} \mathcal{P}_{n}(2), \\
\Upsilon^{\prime \prime}: \Pi\left(\mathcal{H}_{i}\right) \longrightarrow \mathcal{S} \mathcal{P}_{n}(2),
\end{array}\right.
$$

where $\Upsilon^{\prime}, \Upsilon^{\prime \prime}$ are linear maps.

The space $\mathrm{H}^{1}\left(\mathfrak{o s p}(1 \mid 2)_{i}, \mathcal{S} \mathcal{P}_{n}(2)\right), i=1$, 2, determines the linear maps $\Upsilon^{\prime}$. Then $\Upsilon^{\prime}=\Phi^{i}$. More precisely, we get:

Case $1 n=-1, \Upsilon^{\prime}=v_{1} \Phi_{1}^{i}+v_{2} \Phi_{2}^{i}+v_{3} \Phi_{3}^{i}+v_{4} \widetilde{\Phi}_{3}^{i}$, where the coefficients $v_{k}$ are constants.

Case $2 n=0, \Upsilon^{\prime}=v_{5} \Phi_{4}^{i}+v_{6} \Phi_{5}^{i}+v_{7} \Phi_{6}^{i}+v_{8} \widetilde{\Phi}_{6}^{i}$.

In each case, the 1-cocycle conditions determines $\Upsilon^{\prime \prime}$. We obtain for $n=-1, \Upsilon=$ $v_{1} \Upsilon_{1}+v_{3} \Upsilon_{3}$ and for $n=0, \Upsilon=v_{5} \Upsilon_{3}+\left(v_{6}+v_{8}\right) \Upsilon_{4}+v_{7} \Upsilon_{5}+v_{8} \Upsilon_{6}$.

Thus, the space $\mathrm{H}^{1}\left(\mathfrak{o s p}(2 \mid 2), \mathcal{S} \mathcal{P}_{-1}(2)\right)$ is generated by the nontrivial cocycles $\Upsilon_{1}$ and $\Upsilon_{2}$ and the space $\mathrm{H}^{1}\left(\mathfrak{o s p}(2 \mid 2), \mathcal{S} \mathcal{P}_{0}(2)\right)$ is spanned by the nontrivial cocycles $\Upsilon_{3}, \Upsilon_{4}, \Upsilon_{5}$ and $\Upsilon_{6}$.

Theorem 3.7 is proved. 


\section{Corollary 3.3}

$$
\mathrm{H}^{1}(\mathfrak{o s p}(2 \mid 2), \mathcal{S} \Psi \mathcal{D O}(2)) \simeq \mathbb{R}^{6}
$$

The nontrivial spaces $\mathrm{H}^{1}(\mathfrak{o s p}(2 \mid 2), \mathcal{S} \Psi \mathcal{D O}(2))$ are spanned by the cohomology classes of the 1-cocycles $\Theta_{n}$ defined by:

$$
\begin{aligned}
& \Theta_{1}\left(X_{F}\right)=(-1)^{p(F)} \eta_{1} \eta_{2}(F) \xi^{-1} \bar{\zeta}_{1} \bar{\zeta}_{2} \\
& \Theta_{2}\left(X_{F}\right)=F^{\prime} \xi^{-1} \bar{\zeta}_{1} \bar{\zeta}_{2} \\
& \Theta_{3}\left(X_{F}\right)=F^{\prime} \\
& \Theta_{4}\left(X_{F}\right)=(-1)^{p(F)} \eta_{1} \eta_{2}(F) \\
& \Theta_{5}\left(X_{F}\right)=\left((-1)^{p(F)}\left(\bar{\eta}_{1}^{3}(F) \bar{\zeta}_{1}+\bar{\eta}_{2}^{3}(F) \bar{\zeta}_{2}\right)-F^{\prime \prime}\right) \xi^{-1} \\
& \Theta_{6}\left(X_{F}\right)=F^{\prime \prime} \xi^{-2} \bar{\zeta}_{1} \bar{\zeta}_{2}+(-1)^{p(F)}\left(\bar{\eta}_{1}^{3}(F) \bar{\zeta}_{2}-\bar{\eta}_{2}^{3}(F) \bar{\zeta}_{1}\right) \xi^{-1}
\end{aligned}
$$

Proof of Theorem 3.7 Following Ovsienko and Roger in [10], based on the computations of successive differentials of the spectral sequences corresponding to the complex $C^{*}(\mathfrak{o s p}(2 \mid 2), \mathcal{S P}(2))$ to compute the explicit expressions of the basis cocycles: consider a cocycle in $\mathcal{S P}(2)$, but compute its differential as if it were with values in $\mathcal{S} \Psi \mathcal{D O}(2)$ and keep the symbolic part of the result. This gives a new cocycle of degree equal to the degree of the previous one plus one, and its class will represent its image under $d_{1}$. The higher order differentials $d_{k}$ can be calculated by iteration of this procedure, the space $E_{k}^{p+k, q-k+1}$ contains the subspace coming from $H^{p+q+1}\left(\mathfrak{o s p}(2 \mid 2) ; G r^{p+1}(\mathcal{S} \Psi \mathcal{D O}(2))\right)$, where $G r^{p}(\mathcal{S} \Psi \mathcal{D O}(2))$ is isomorphic, as $\mathfrak{o s p}(2 \mid 2)$-module to the space of weighted densities $\mathfrak{F}_{p}^{2}$. We give explicit expressions of the basis cocycles.

Acknowledgements We would like to thank Mabrouk Ben AMMAR and Nizar Ben FRAJ for helpful discussions.

Open Access This article is distributed under the terms of the Creative Commons Attribution Noncommercial License which permits any noncommercial use, distribution, and reproduction in any medium, provided the original author(s) and source are credited.

\section{References}

1. Agrebaoui, B., Ben Fraj, N.: On the cohomology of Lie superalgebra of contact vector fields on $S^{1 \mid 1}$. Bulletin de la Scociété Royale des Sciences de Liège 72(6), 365-375 (2004)

2. Agrebaoui, B., Ben Fraj, N., Omri, S.: On the cohomology of Lie Superalgebra of contact vector fields on $S^{1 \mid 2}$. J. Nonlinear Math. Phys. 13, 523-534 (2006)

3. Basdouri, I., Sayari, E.: On the cohomology of the Orthosymplectic superalgebra. Acta Math. Hung. 130(1-2), 155-166 (2011)

4. Ben Fraj, N., Omri, S.: Deforming the Lie superalgebra of contact vector fields on $S^{1 \mid 1}$. J. Nonlin. Math. Phys. 13(1), 19-33 (2006). math-ph/0603058

5. Ben Fraj, N., Omri, S.: Deformating the Lie superalgebra of contact vector fields on $S^{1 / 2}$ inside the Lie superalgebra of pseudodifferential operators on $S^{1 / 2}$. Theor. Math. Phys. 163(2), 618-633 (2010)

6. Fialowski, A.: An example of formal deformations of Lie algebras. In: Proceedings of NATO Conference on Deformations Theory of Algebras, Kluwer, pp. 3 (1988)

7. Fuchs, D.B.: Cohomology of Infinite-Dimensional Lie Algebras. Plenum Publ., New York (1986) 
8. Grozman, P., Leites, D., Shchepochkina, I.: Lie superalgebras of string theories. Acta Math. Vietnam. 26(1), 27-63 (2001). arXiv: hep-th/9702120

9. Ovsienko, V., Roger, C.: Deforming the Lie algebra of vector fields on S1 inside the Lie algebra of pseudodifferential symbols on S1. arXiv:math/9812074v1 [math.QA] (1998) 11 Dec 1998

10. Ovsienko, V., Roger, C.: Deforming the Lie algebra of vector fields on $S^{1}$ inside the Poisson algebra on $\dot{T}^{*} S^{1}$. Commun. Math. Phys. 198, 97-110 (1998) 